Research Article

\title{
Identification of a Modified HOXB9 mRNA in Breast Cancer
}

\author{
Ayako Nakashoji, Tetsu Hayashida (D), Yuko Kawai, Masayuki Kikuchi, Rurina Watanuki, \\ Takamichi Yokoe, Tomoko Seki, Maiko Takahashi, Kazuhiro Miyao, Shigeo Yamaguchi, \\ and Yuko Kitagawa
}

\author{
Department of Surgery, Keio University School of Medicine, Shinanomachi 35 Shinjuku-ku, Tokyo 160-0016, Japan \\ Correspondence should be addressed to Tetsu Hayashida; tetsu@keio.jp
}

Received 3 August 2019; Revised 29 December 2019; Accepted 7 January 2020; Published 13 February 2020

Academic Editor: Rossana Berardi

Copyright (c) 2020 Ayako Nakashoji et al. This is an open access article distributed under the Creative Commons Attribution License, which permits unrestricted use, distribution, and reproduction in any medium, provided the original work is properly cited.

\begin{abstract}
First identified as a developmental gene, HOXB9 is also known to be involved in tumor biological processes, and its aberrant expression correlates with poor prognosis of various cancers. In this study, we isolated a homeodomain-less, novel HOXB9 variant $(H O X B 9 v)$ from human breast cancer cell line-derived mRNA. We confirmed that the novel variant was produced from variationless HOXB9 genomic DNA. RT-PCR of mRNA isolated from clinical samples and reanalysis of publicly available RNAseq data proved that the new transcript is frequently expressed in human breast cancer. Exogenous HOXB9v expression significantly enhanced the proliferation of breast cancer cells, and gene ontology analysis indicated that apoptotic signaling was suppressed in these cells. Considering that HOXB9v lacks key domains of homeobox proteins, its behavior could be completely different from that of the previously described variationless HOXB9. Because none of the previous studies on HOXB9 have considered the presence of HOXB9v, further research analyzing the two transcripts individually is warranted to re-evaluate the true role of HOXB9 in cancer.
\end{abstract}

\section{Introduction}

Homeobox (HOX) genes were initially characterized as developmental genes, which code for transcription factors that play critical roles in embryogenesis. Evolutionarily, they are highly conserved and share a high degree of homology, especially within the same paralog groups. All 39 mammalian HOX genes consist of two exons and a single intron. The homeobox domain, encoded in the second exon [1], includes the DNA binding site. The diverse and specific transcriptional activities of the HOX proteins often depend on key cofactors including PBX, MEIS, and PREP, which interact with hexapeptide motifs of HOX proteins [2].

HOX genes play key roles in both solid and hematological malignancies, including cancers of the colon, breast, prostate, lung, brain, thyroid, ovary, bladder, kidney, skin, and blood $[3,4]$. HOXB9, the ninth paralog in the HOX-B cluster, is associated with the growth and progression of multiple cancers. In lung adenocarcinoma, HOXB9 promotes metastasis by activating the WNT signaling pathway $[5,6]$. In breast cancer, the gene induces the expression of proangiogenic factors, increasing the cell motility and supporting epithelial-mesenchymal transition (EMT) $[7,8]$. HOXB9 also promotes the growth of colon cancer by activating IL6 signaling, inducing the secretion of angiogenic factors and increasing proliferation of tumor cells [9]. Similar observations are found in ovarian cancer and hepatocellular carcinoma $[10,11]$. Thus, HOXB9 activates the WNT signaling pathway and enhances the acquisition of capabilities critical to the transformation of normal cells to cancer, including EMT and the growth of new vasculature within the tumor microenvironment.

HOXB9 is also directly associated with cancer-induced patient mortality. The duration of disease-free and overall survival of patients with HOXB9-positive breast cancer is significantly shorter compared with patients with HOXB9negative breast cancer [12]. Increased HOXB9 expression significantly correlates with decreased overall survival for 
patients with colorectal cancer [9]. Patients of laryngeal squamous cell carcinoma, hepatocellular carcinoma, glioma, and endometrial cancer also present poor outcomes or tumor progression, resulting from aberrant HOXB9 expression [13-16].

The recent elucidation of the critical and diverse roles HOXB9 plays in various cancers have led us to explore the mechanism of this gene's role in cancer progression. In the present study, we identified and characterized a HOXB9 variant $(H O X B 9 v)$ of mRNA from human breast cancer cell lines. The sequence of $H O X B 9 v$ largely differs from the previously known $H O X B 9$ normal transcript (HOXB9n), and we found it lacks some important domains of HOX genes. Based on these findings, we inferred its role and function were different from HOXB9n. This study aimed to confirm the presence of $\mathrm{HOXB9v}$ in clinical breast cancer specimens and investigate the role of $\mathrm{HOXB9v}$ in breast cancer progression.

\section{Materials and Methods}

2.1. Cell Culture. We cultured eight human breast cancer cell lines (MCF7, MDA-MB-231, MDA-MB-468, Hs578T, HCC38, BT-474, BT-549, and SKBR3), a human colon cancer cell line (WiDR), and a mouse breast cancer cell line (4T1) in DMEM supplemented with 10\% FBS (Thermo Fisher Scientific, Waltham, MA, USA) with the addition of antibiotic and antimycotic agent (antibiotic-antimycotic mixed stock solution, Nacalai Tesque, Inc., Kyoto, Japan). T47D cells were grown in RPMI1640 medium with 10\% FBS and antibiotic and antimycotic agents. MCF10A cells were grown in DMEM/F12 (1:1) medium with 5\% horse serum (Thermo Fischer Scientific), $20 \mathrm{ng} / \mathrm{mL}$ EGF (PeproTech, Rocky Hill, NJ, USA), $0.5 \mathrm{mg} / \mathrm{mL}$ hydrocortisone, $100 \mathrm{ng} /$ $\mathrm{mL}$ cholera toxin, $10 \mu \mathrm{g} / \mathrm{mL}$ insulin (all Sigma-Aldrich, St. Louis, MO, USA), and antibiotic and antimycotic agents. Cells were maintained at $37^{\circ} \mathrm{C}$ in a humidified $5 \% \mathrm{CO}_{2}$ incubator.

2.2. Patients and Samples. Clinical specimens of human breast cancer $(n=14)$ were collected from patients with primary operable breast cancer who underwent total or partial mastectomy between July and November 2018 in Keio University Hospital (Tokyo, Japan). Patient-matched healthy breast epithelium samples $(n=6)$ were collected from healthy breast tissue from patients who underwent total mastectomy. Ethics approval for the present study was provided by the Ethics Committee at the Keio University School of Medicine (approval number: 20180090), and the study was performed in accordance with the provisions of the Declaration of Helsinki (as revised in Fortaleza, Brazil, October 2013). All included patients gave informed consent.

2.3. $m R N A$ and Genomic DNA Extraction from Cell Lines and Clinical Specimens. Total RNA and genomic DNA were extracted using the RNeasy Mini Kit and QIAmp DNA Mini Kit (Qiagen, Hilden, Germany) following the manufacturer's instructions. Clinical specimens were homogenized using a
Minilys homogenizer (Bertin Instruments, Bretonneux, France) using $2.4 \mathrm{~mm}$ metal beads prior to mRNA extraction. Total RNA was converted to cDNA using the HighCapacity RNA-to-cDNA Kit (Thermo Fischer Scientific).

2.4. HOXB9 Cloning. HOXB9 transcripts and genomic DNA were amplified by PCR and subsequently cloned into the pME-HA vector (Lucigen, Middleton, WI, USA) using the Expresso CMV Cloning \& Expression system (Lucigen). The primers used were as follows: sense $5^{\prime}$ GAAGGAGATACCACCATGTCCATTTCTGGGACGCTTAGC $3^{\prime}$ and antisense $5^{\prime}$ GGGCACGTCATACGGATACTCTTTGCCC TGCTCCTTATT $3^{\prime}$.

After transformation into Competent Quick DH5 $\alpha$ cells (Toyobo, Osaka, Japan) and culturing in kanamycin-containing LB plates, at least 4 colonies were selected for each sample.

2.5. Sequence Analysis. Sequences were analyzed using the BigDye Terminator V3.1 Cycle Sequencing kit (Thermo Fisher Scientific) and Applied Biosystems 3500 Genetic analyzer (Thermo Fisher Scientific). The GENETYX-MAC Ver.19 software (GENETYX, Osaka, Japan) was used for homology alignment.

2.6. RT-PCR Analysis. The expressions of HOXB9n and $H O X B 9 v$ were detected by RT-PCR using the following primers: sense, 5' TGTCCATTTCTGGGACGCTT 3'; antisense, 5' CTACGGTCCCTGGTGAGGTA 3'. The genomic DNA of HOXB9 was detected by PCR using the following primers: sense, $5^{\prime}$ CGAGAGAGCTGCAAGTCGAT $3^{\prime}$; antisense, 5' CTGCCGTCCGTCTACCAC 3'. The primers for genomic DNA were designed against exon 1 and the intron region of the $H O X B 9$ gene to ensure that the pair will specifically amplify only genomic DNA and not cDNA derived from mRNA. The conditions applied for amplification were as follows: $94^{\circ} \mathrm{C}$ for 1 minute, followed by 35 cycles at $95^{\circ} \mathrm{C}$ for 5 seconds, $55^{\circ} \mathrm{C}$ for 5 seconds, and $72^{\circ} \mathrm{C}$ for 5 seconds, and run on the Life ECO thermal cycler (Hangzhou Bioer Technology, Hangzhou, China) using the SapphireAmp Fast PCR Master Mix (Takara Bio, Shiga, Japan) or KOD-Plus-Neo (Toyobo).

2.7. Public Data Reanalysis. The RNA sequence data set (GSE119937) in FASTQ format was downloaded via SRA (SRP161704) using the SRA Toolkit (version 2.3.4-2). RNAseq reads were aligned by STAR (version 2.6.1b) against the hg38 reference genome. All reads mapped on the HOXB9 gene were visually confirmed by taking snapshots in IGV (version 2.4.15).

\subsection{Establishment of Stable MCF7 Cell Lines Overexpressing} $H O X B 9 n$ or HOXB9v. The HOXB9n and HOXB9v sequences were amplified by PCR and subsequently cloned into the pBiT3.1-N [CMV/HiBiT/Blast] expression vector (Promega, Tokyo, Japan) at the Xhol and BamHI sites using 
Ligation high Ver.2 (Toyobo). The primers used for amplification were as follows: sense, $3^{\prime}$ ATACCTCGAGG TCCATTTCTGG $5^{\prime}$; antisense, $3^{\prime}$ CACGTCATACGGAT CCTCTTTG $5^{\prime}$. MCF7 cells were transfected with the HiBiT-tagged HOXB9n or HOXB9v vector using the Viafect Transfection reagent (Promega) and selected with $10 \mu \mathrm{g} / \mathrm{mL}$ of blasticidin for at least 4 weeks. The expression of HiBiTtagged HOXB9n and HOXB9v proteins was detected using the Nano-Glo HiBiT Blotting System (Promega) as per the manufacturer's instructions.

2.9. Transient Overexpression of HOXB9n and HOXB9v in MDA-MB-468 Cells. MDA-MB-468 cells were transfected with aforementioned HiBiT-tagged HOXB9n or HOXB9v vector using the jetPRIME (Polyplus-transfection, Illkirch, France) as per the manufacturer's instructions.

2.10. Quantitative Real-Time PCR. Quantitative real-time PCR was run on ViiA7 (Thermo Fisher Scientific) using Fast SYBR Green Master Mix (Thermo Fisher Scientific). Preincubation was performed for 20 seconds at $95^{\circ} \mathrm{C}$ and amplification for 41 cycles ( 1 second of denaturation at $95^{\circ} \mathrm{C}$ and 20 seconds of annealing and extension at $60^{\circ} \mathrm{C}$ ), followed by melt-curve analysis. GAPDH served as an internal control, and QuantStudio Real-Time PCR Software v1.2 (Thermo Fisher Scientific) was used for quantification. The relative standard curve method was used for linear regression analysis of unknown samples, and data are presented as fold change between samples. The primers used were as follows: HOXB9: sense, $5^{\prime}$ CGGTGGCTGTCGTGAAATT 3'; antisense, 5' CGAGACAATCACCCCCAAAG 3'; GAP $D H$ : sense, $5^{\prime}$ ATCATCCCTGCCTCTACTGG $3^{\prime}$; antisense, $5^{\prime}$ TTTCTAGACGGCAGGTCAGGT 3'.

2.11. Cell Proliferation Assay. Cell proliferation in threedimensional (3D) culture was measured using the 24-well Bio-Assembler kit and NanoShuttle-PL (Greiner Bio-One, Kremsmünster, Austria). Further, 20,000 cells/well were incubated for 48 hours before taking photomicrographs.

Cell proliferation in flat culture was measured using Cell Count Reagent SF (Nacalai Tesque). Briefly, 5000 cells/well in 96-well microtiter plates (Sumilon, Sumitomo Bakelite, Tokyo Japan) were incubated for 5 days or after 24 hour continuous exposure to either $30 \mathrm{nM}$ HXR9 or $30 \mathrm{nM}$ CXR9 for 4 days. The absorbance in the wells was measured on days 1,3 , and 5 using a Sunrise Rainbow-RC (TECAN, Männedorf, Switzerland) microplate spectrophotometer at $450 \mathrm{~nm}$, using $600 \mathrm{~nm}$ as reference. The HXR9 (WYPWMKKHHRRRRRRRRR-) and control CXR9 (WYPAKKHHRRRRRRRRR) peptides were synthesized by Eurofins Genomics K. K. (Tokyo, Japan).

2.12. Microarray and Differential Expression Analyses. Total RNA was isolated using the RNeasy Mini kit (Qiagen). Microarray was performed using the human Clariom S assay (Thermo Fisher Scientific) by the GeneChip Scanner 3000 7G system (Affymetrix, Santa Clara, CA, USA), and the results were analyzed by TAC 4.0 software (Thermo Fisher Scientific). Genes having a false discovery rate (FDR) under 0.05 and upregulated in HOXB9v samples were considered as upregulated differentially expressed genes (up-DEGs). DAVID Bioinformatics Resources 6.8 [17] was used for gene ontology and pathway analysis of DEGs. R ver. 3.5.0 software was used to draw the heatmap of 37 genes included in GO: 0043069 (negative regulation of programmed cell death).

\section{Results}

3.1. Identification of a Novel Transcript HOXB9v, Which Lacks Important Domains of HOX Gene. We isolated total RNA from human breast cancer cell lines (MCF7, T47D, MDAMB-231, MDA-MB-468, HCC38, BT-474, BT-549, Hs578T, and SKBR3), a normal human mammary gland cell line (MCF10A), and a human colon cancer cell line (WiDR). We cloned and sequenced the HOXB9 gene from the nucleic acids derived from these cell lines. We isolated a novel variant of the HOXB9 transcript from the MCF7, T47D, MDA-MB-231, MDA-MB-468, Hs578T, HCC38, and MCF10A cell lines. The sequence homology of the new transcript is shown in Figures 1(a) and S1.

We will refer to the new truncated transcript as $H O X B 9 v$, to distinguish from the full-length $H O X B 9$ transcript $(H O X B 9 n)$. A 100-base deletion in exon 1 in the new transcript leads to a frameshift and the formation of a stop codon (TAG), which truncates the protein coding at AA167. Figure 1(b) is a schematic diagram of HOXB9 transcripts showing the exons and the deleted lesion. The encoded protein will therefore possibly lack the homeobox domain, DNA binding domain, and the hexapeptide motif, a major player in cofactor interactions (Figure 1(c)). The HOXB9v sequence has been submitted to GenBank under Accession No. LC466645.

We next performed PCR analyses to verify the presence of the HOXB9v transcript and identify genomic DNA variations of the HOXB9 gene in human breast cancer cell lines. The primer target region included the deletion site of $H O X B 9 v$ and the amplicon for HOXB9n was $643 \mathrm{bp}$ and $543 \mathrm{bp}$ for HOXB9v. We detected both $H O X B 9 n$ and $H O X B 9 v$ transcripts in breast cancer cell lines (Figure 2(a)). The PCR products were sequenced and were confirmed that each band corresponded to the exact sequence of the HOXB9n or HOXB9v (Figure 2(b)). By PCR of breast cancer cell lines' genomic DNA, no bands indicative of genomic DNA variations in $H O X B 9$ were detected. The amplicon of variation-less HOXB9 genomic DNA was $569 \mathrm{bp}$ (Figure 2(c)). Sequencing of the PCR products confirmed that they had no variations or deletions in genomic DNA (Figure S2). These findings show that the two transcripts (HOXB9n and $H O X B 9 v$ ) were produced from variation-less HOXB9 genomic DNA.

To determine the presence of $H O X B 9 v$ transcripts in human breast cancer samples, we performed PCR analysis. $H O X B 9 v$ was commonly detected from clinical breast cancer samples (Figure 3(a)), regardless of their hormone receptor and HER2 status. However, HOXB9v was not detected in normal mammary gland samples (Figure 3(b)). 


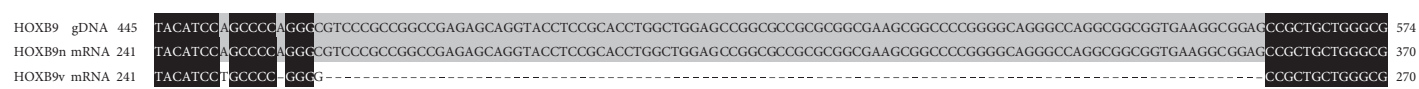

(a)

Exon I

Exon II
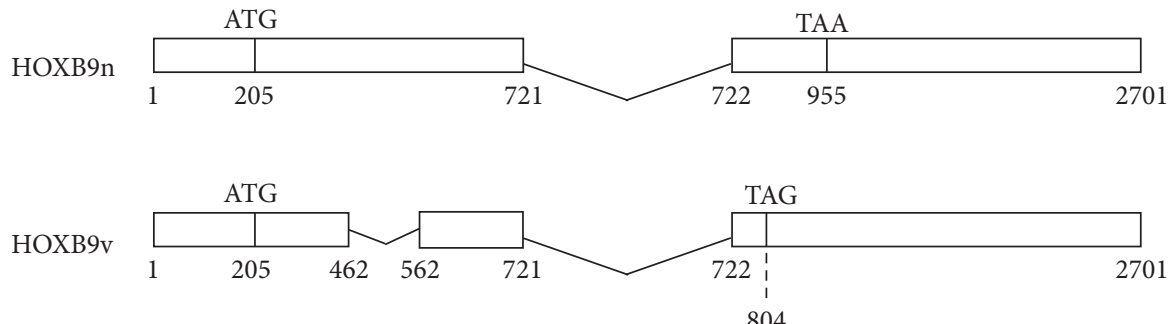

(b)

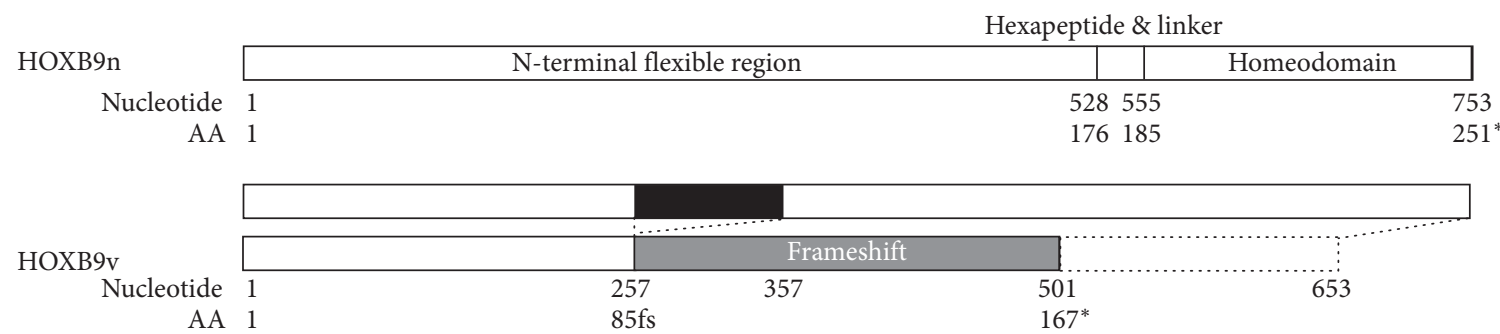

(c)

Figure 1: Structure and sequence of HOXB9n and HOXB9v. (a) Sequences of genomic DNA HOXB9, mRNA HOXB9n, and mRNA HOXB9v (black and grey highlighting indicates homology between sequences). (b) Schematic diagram of HOXB9n (upper) and HOXB9v (lower) transcripts showing the exons, splicing regions, and the deleted region. In HOXB9v, a 100 bp deletion in exon 1 leads to a frameshift and a stop codon formation (TAA). (c) Protein structure of HOXB9n and HOXB9v. Transcription from the start codon (ATG) to the stop codon (TAA) results in the translation of a full-length HOXB9n protein (upper). The $100 \mathrm{bp}$ deletion in HOXB9v (shown in black) leads to a frameshift from AA85 (shown in grey) and truncation of protein coding by a stop codon (TAG) at AA167, which results in HOXB9v protein without hexapeptide and homeodomain. ${ }^{*}$ Stop codon.

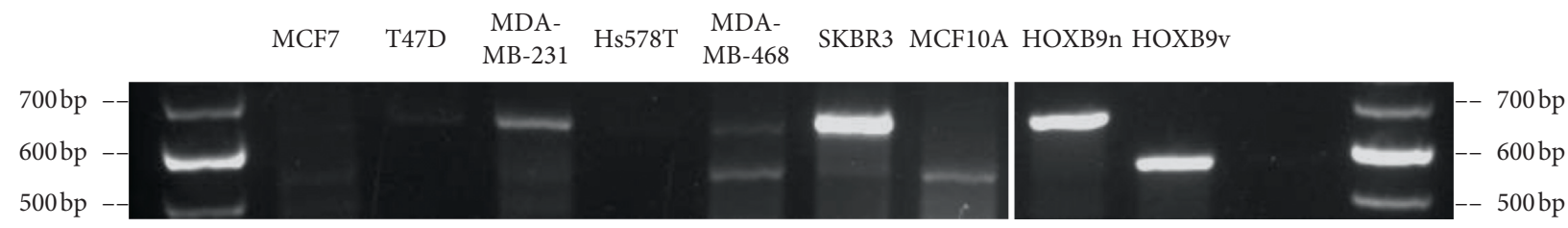

(a)

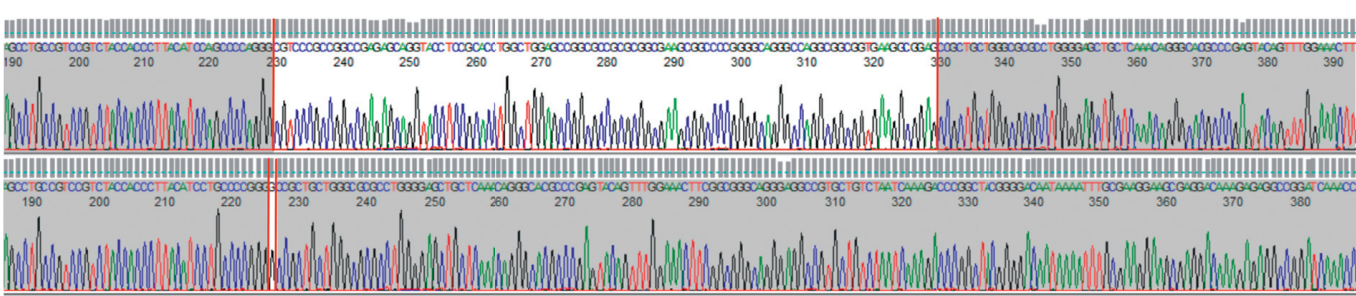

(b)

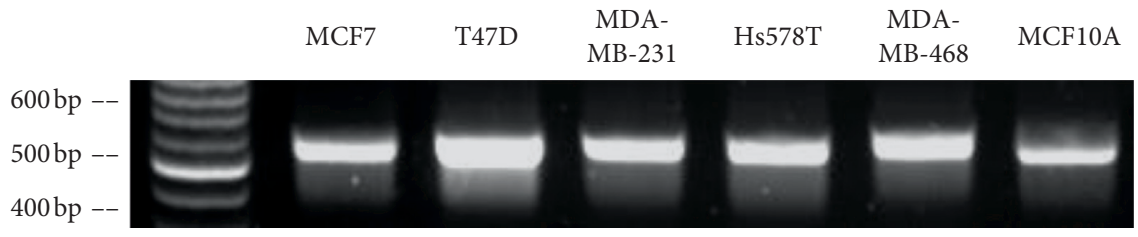

(c)

FIgURE 2: Detection of mRNA and genomic DNA of HOXB9 in cell lines. (a) Both HOXB9n and HOXB9v transcripts were detected in breast cancer cell line mRNA (cDNA). (b) Sequencing confirmation of PCR product of Figure 2(a); SKBR3 (HOXB9n, upper column) and MCF10A (HOXB9v, lower column). (c) No genomic variation was detected in genomic DNA of breast cancer cell line genomic DNA. $H O X B 9 v$ transcripts are commonly found in human breast cancer specimens. 


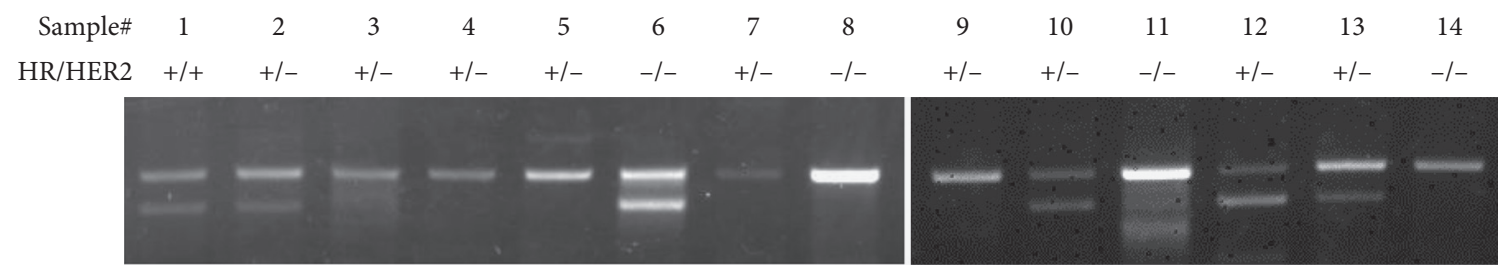

(a)

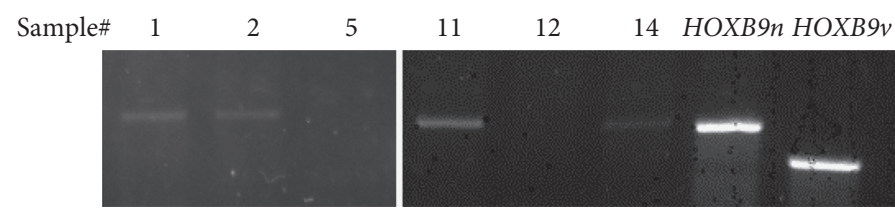

(b)

FIgURE 3: Detection of HOXB9v in clinical samples. (a) HOXB9n and HOXB9v were detected in breast cancer clinical samples. (b) HOXB9v was not detected in normal mammary gland samples. Hormone receptor status and HER2 status of each cancer sample are shown beneath each sample number. We assigned the same sample number if cancer and normal gland samples were acquired from the same patient.

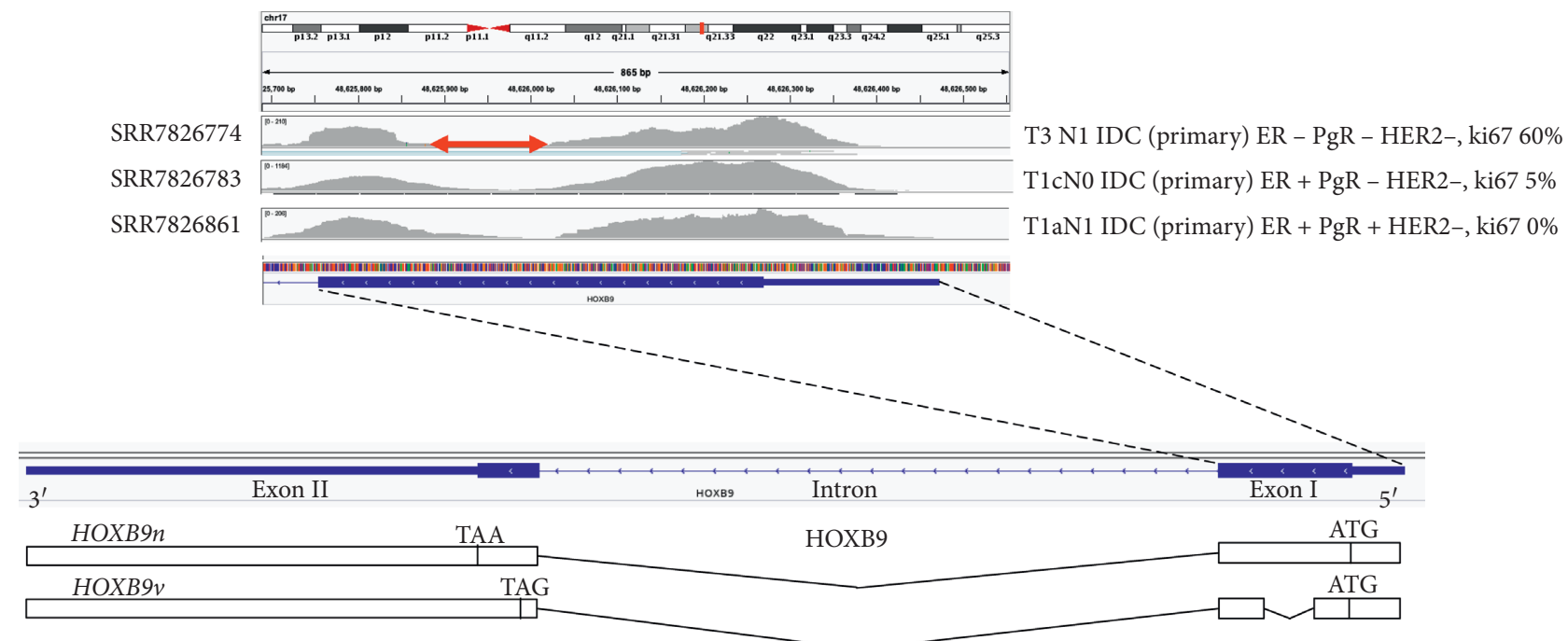

FIGURE 4: NGS data indicates the presence of HOXB9v in breast cancer. Showing RNA sequence breast cancer sample data (SRR782677) mapped on HOXB9 exon 1. The blue band on the bottom shows the coding region (the thick part) and the $5^{\prime}$ UTR (the thin part) of exon 1. There is a region where data are sparsely mapped (indicated with the red arrow), which matches the deletion region in $H O X B v$. HOXB9v overexpressing MCF7 and MDA-MB-468 cells proliferated faster compared with HOXB9n overexpressing cells.

Thus, to further confirm the presence of $H O X B 9 v$ in human breast cancer samples, we reanalyzed a publicly available breast cancer RNA sequence data set (GSE119937) [18] and mapped the reads onto the HOXB9 gene sequence. We identified a region in exon 1 where the number of mapped reads was low in numerous samples; this region matched the deletion region of HOXB9v (Figure 4).

To determine the role of HOXB9v in breast cancer, we established stable MCF7 cell lines overexpressing HOXB9n or HOXB9v. Gene and protein expressions of HOXB9n and HOXB9v were verified in both cell lines (Figures 5(a) and $5(\mathrm{~b}))$. Cell proliferation assays in both 3D culture and flat culture showed that $\mathrm{HOXB9v}$ overexpression increased MCF7 cell growth (Figures 5(c) and 5(d)). We also transiently overexpressed HOXB9n or HOXB9v in MDAMB-468 cells, and the cell proliferation assay showed that HOXB9v overexpression increased MDA-MB468 cell growth (Figures 5(e) and 5(f)).

3.2. HXR9 and CXR9 Treatment. HXR9, an 18-amino acid peptide, competently inhibits the hexapeptide motif of HOX proteins and prevents HOX-PBX binding [19]. HOXB9n and HOXB9v expressing MCF7 cells were treated with HXR9 or a control peptide, CXR9 (Figures 6(a) and 6(b)). Although HOXB9v lacks the hexapeptide motif, which is known to interact with PBX proteins [20], HXR9 significantly inhibited proliferation of both cell lines. 


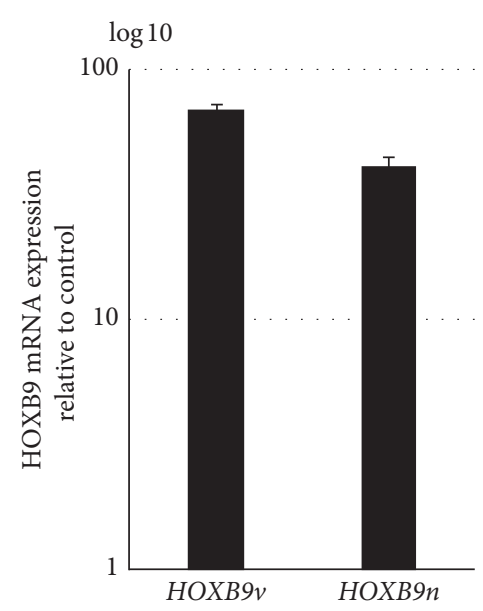

(a)

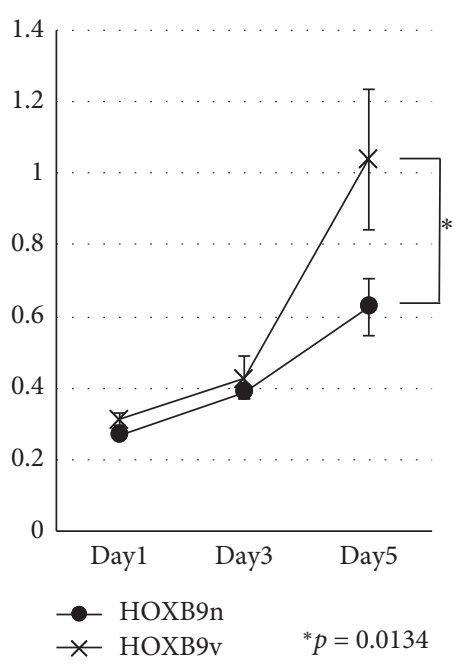

(d)

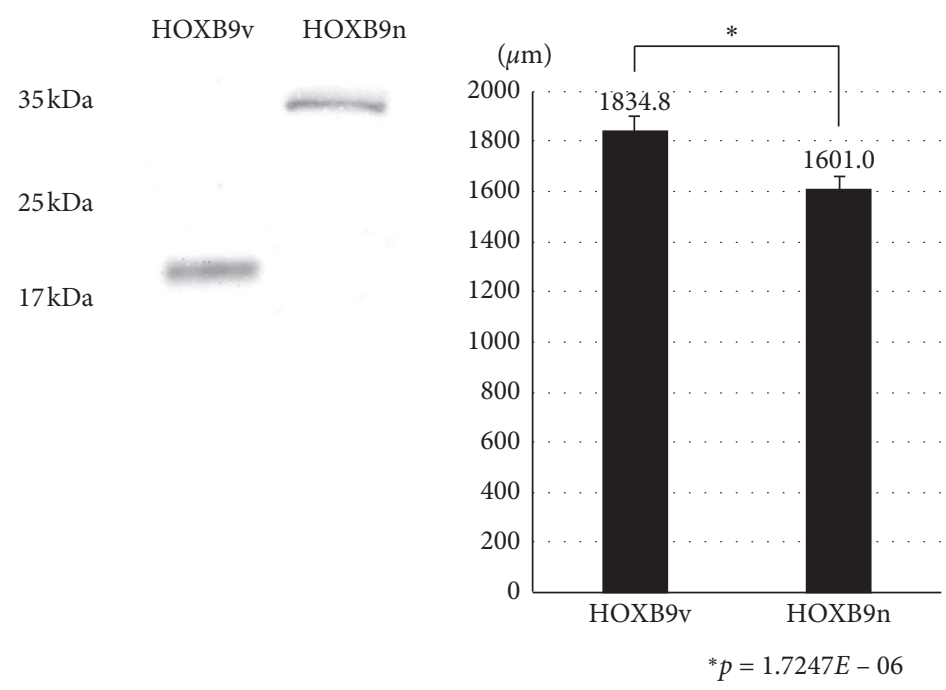

(b)

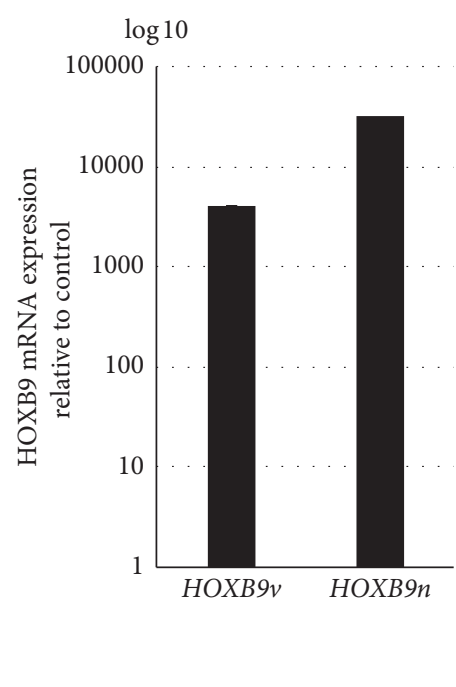

(e)

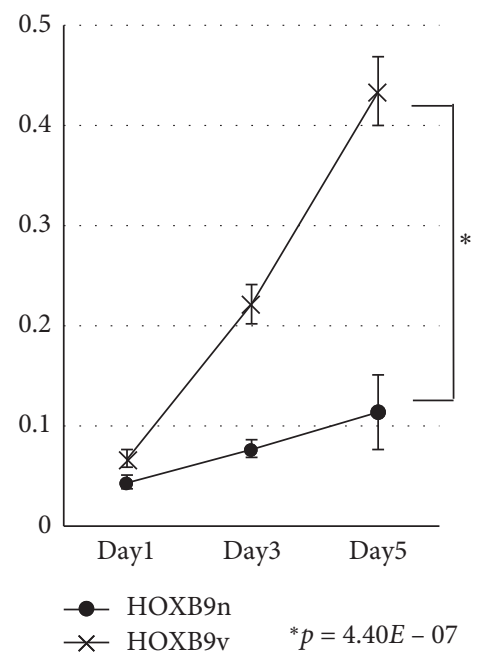

(f)

Figure 5: (a-d) Confirmation and proliferation of stable MCF7 cell lines that overexpress HOXB9n or HOXB9v. Levels of HOXB9n and HOXB9v (a) mRNA and (b) protein in stable cell lines. Proliferation of the HOXB9n and HOXB9v stable cell lines using a (c) 3D cell culture model and a (d) 2D cell culture model. (e-f) Confirmation and proliferation of MDA-MB-468 cells with transient overexpression of HOXB9n or HOXB9v. (e) Levels of HOXB9n and HOXB9v mRNA expression. (f) Proliferation of HOXB9n and HOXB9v overexpressing cells by $2 \mathrm{D}$ cell culture model.

3.3. Microarray and Gene Ontology Analysis. To explore the reason behind the faster proliferation of HOXB9v overexpressing cells, we performed microarray and gene ontology analyses using HOXB9n and HOXB9v-expressing MCF7 cells. We chose 1056 genes as DEGs, and gene ontology analysis showed that up-DEGs between HOXB9n and HOXB9v expressing cells presented significant differences in pathways relevant to apoptosis suppression (GO:0060548 negative regulation of cell death, GO:0043069 negative regulation of programmed cell death, and GO:0043066 negative regulation of apoptotic process) and steroid hormone response (GO:004 8545 response to steroid hormone, GO:0032870 cellular response to hormone stimulus, and GO:0071383 cellular response to steroid hormone stimulus) (Table 1). The genomic expression heatmap comparing 37 genes involved in the apoptotic process (GO:0043069 negative regulation of programmed cell death) shows that apoptosis is highly suppressed in HOXB9v expressing cells (Figure 7).

\section{Discussion}

In the present study, we identified a novel modified transcript of HOXB9, in which a deletion in exon 1 causes a frameshift, formation of a stop codon, and truncation of protein coding. This leads to a defect in the homeodomain and hexapeptide regions, which are both crucial for HOX gene function. We confirmed that $H O X B 9 v$ is widely present in human breast cancer cell lines and clinical breast cancer samples, however not 


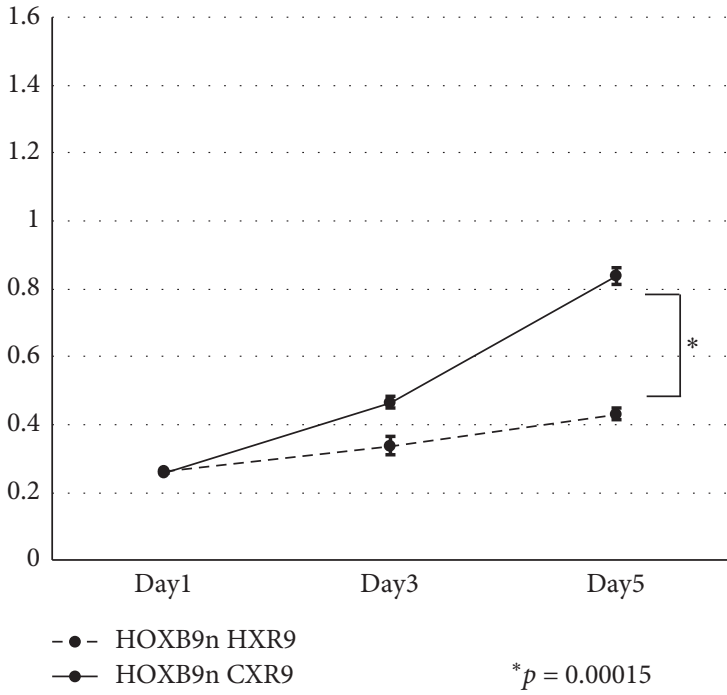

(a)

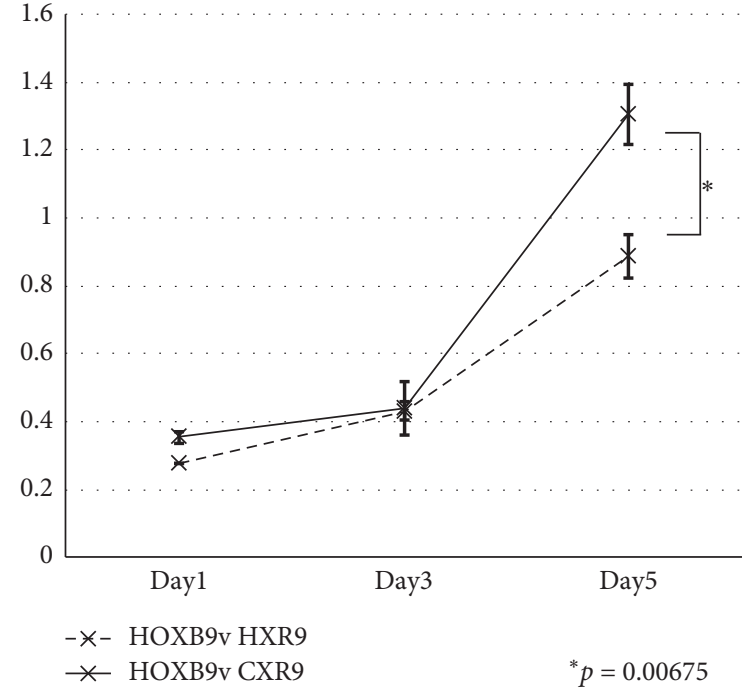

(b)

FIGURE 6: (a) HOXB9n-MCF7 cells and (b) HOXB9v-MCF7 cells were treated with HXR9 or with a control peptide, CXR9. HXR9 significantly inhibited the proliferation of both cell lines.

TABLE 1: Gene ontology analysis by differential gene expression. Apoptosis was suppressed in HOXB9v-MCF7 cells compared with HOXB9n-MCF7 cells.

\begin{tabular}{llc}
\hline Term & $P$ value & Bonferroni \\
\hline Negative regulation of cell death & $7.36 E^{-07}$ & 0.002961 \\
Response to steroid hormone & $2.24 E^{-06}$ & 0.008994 \\
Cellular response to zinc ion & $2.66 E^{-06}$ & 0.01065 \\
Negative regulation of programmed cell death & $3.30 E^{-06}$ & $6.93 E^{-06}$ \\
Cellular response to hormone stimulus & $7.26 E^{-06}$ & 0.013228 \\
Negative regulation of apoptotic process & $1.15 E^{-05}$ & 0.027551 \\
Cellular response to steroid hormone stimulus & $1.17 E^{-05}$ & 0.028853 \\
Negative regulation of transcription, DNA-template & 0.045436 \\
\hline
\end{tabular}

in normal gland samples. Detection of $\mathrm{HOXB9v}$ in MCF10A may be attributed to the fact that this cell line is not karyotypically normal, while it maintains major characteristics of normal breast epithelium [21]. We further confirmed that $H O X B v$ and the previously known HOXB9n were both produced from variationless $H O X B 9$ genomic DNA. Two distinct mRNA products from a single variationless genomic DNA may indicate that $H O X B 9 v$ is a novel splice variant of $H O X B 9$. It may be assumed that $H O X B 9 v$ has a typical splice site of GU as a $5^{\prime}$ donor site and AG as a $3^{\prime}$ acceptor site [22]; however, the single-nucleotide transitions at 248 and 258 and the singlenucleotide deletion at 254 must be taken into account. Further investigation and discussions are needed to decide whether $H O X B 9 v$ is a splice variant or an mRNA modified by a different mechanism. Nonetheless, the modified transcript is certainly not a result of variations in genomic DNA. Additionally, $H O X B 9 v$ results in a different stop codon site from that of $H O X B 9 n$ and may become a target of nonsense-mediated mRNA decay (NMD), an mRNA surveillance mechanism that eliminates premature translation-termination codons. However, NMD is known to be initiated if an exon-junction complex is present more than $50-55$ bases downstream of the stop codon [23]. HOXB9v forms its exon-junction complex upstream from its stop codon; therefore, we infer it escapes from NMD.

Nevertheless, the production of splice variants of HOX genes lacking the homeodomain is likely a common phenomenon. Murine Hoxb9, which shares sequence similarity with human HOXB9, is reported to generate a splice variant without a homeodomain. Other HOX genes, including human HOXA1, HOXB6, HOXA9, HOXA10, murine Hoxb6 and Meis1, and Xenopus XlHbox2, have also been reported to generate splice variants lacking homeodomains [24-28]. Interestingly, HOXA9T, a homeodomain-less isoform of HOXA9, which is structurally similar to $H O X B 9 v$, has been demonstrated to act as an oncogene in leukemia without directly binding to DNA [24]. HOXB9 has been reported to promote tumorigenesis in various types of cancers; however, no previous research on HOXB9 has shown a mRNA variant of HOXB9. Considering that the structure of the HOXB9v protein differs from that of the previously studied HOXB9n protein and lacks important domains such as homeodomain and hexapeptide motif, its function is likely to differ from that of HOXB9n. Further study is required to re-evaluate the role of HOXB9 in 


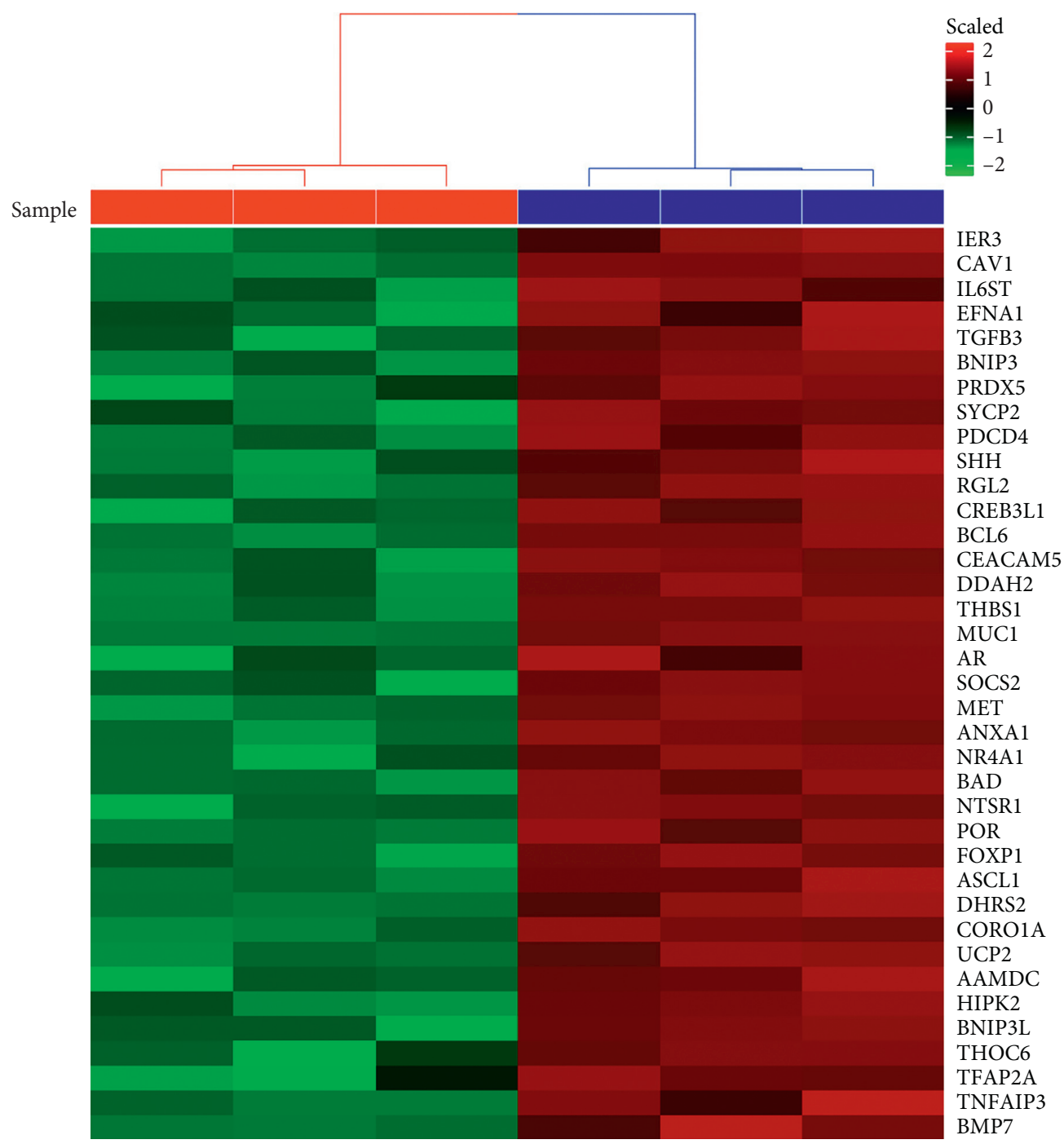

Sample

HOXB9n

HOXB9v

FIgURE 7: Microarray data heatmap of HOXB9n-MCF7 and HOXB9v-MCF7. Genomic heatmap compares expression of 37 genes which are involved in suppression of apoptotic process (GO:0043069), with red and green color intensities indicating high and low expressions, respectively. Programmed cell death is suppressed in HOXB9v-MCF7 cells.

cancer. HOXB9n and HOXB9v should be assessed separately.

In the growth assay, MCF7 and MDA-MB-468 cells expressing HOXB9v at high levels presented more rapid proliferation than did those expressing HOXB9v. It is meaningful that similar results were observed in two cell lines: one with hardly any expression of HOXB9n and HOXB9v (MCF7) and the other with high expression of both (MDA-MB-468). Fostered proliferation by HOXB9v may be attributed to the suppressed apoptosis observed in our microarray studies. Gene ontology analysis of HOXB9v-expressing cells compared to HOXB9nexpressing cells indicated significant upregulation of pathways related to apoptosis suppression, further underscoring its role in this regard. Several HOX genes have been reported to regulate apoptosis in cancer $[29,30]$, and HOX-regulated apoptosis is a general mechanism used during development to maintain metameric patterns [31, 32]. However, the role of HOXB9 in apoptosis is yet to be investigated.

The binding selectivity of HOX proteins is influenced by cofactors, including members of the PBX, MEIS, and PREP families [2]. Additionally, HOX-PBX interactions involve a short HOX protein motif, the hexapeptide, located upstream of the homeodomain [33]. HXR9 is an 18-amino acid peptide and suppresses tumor proliferation by inhibiting HOX-PBX binding [19]. In our proliferation assay, HOXB9v expressing cell lines were sensitive to the HOX-PBX inhibitor HXR9, even though HOXB9v lacks the hexapeptide. This may be because HOXB9v, in consort with HOXB9n or 
other HOX proteins, indirectly promotes HOX-PBX binding.

\section{Conclusions}

We report a modified $H O X B 9$ mRNA variant that results in a homeodomain defect. We confirmed its presence in human breast cancer cell lines and breast cancer clinical samples and also revealed that HOXB9v may promote breast cancer proliferation by suppression of apoptosis. Further research is warranted to analyze HOXB9v and HOXB9n individually and re-evaluate the true role of HOXB9 in cancer.

\section{Data Availability}

The HOXB9v sequence has been submitted to GenBank under Accession No. LC466645. The RNA sequence data set (GSE119937) used in public data reanalysis in the fastq format is available via SRA (SRP161704). Other datasets used and/or analyzed during the current study are available from the corresponding author on reasonable request.

\section{Conflicts of Interest}

The authors declare that there are no conflicts of interest regarding the publication of this paper.

\section{Acknowledgments}

The authors greatly thank Dr. Sasaki Takafumi, Keio University School of Medicine, for technical advice. The authors also thank Dr. Shunsuke Kato for scientific advice and Mr. Kazuya Takakuwa for help in bioinformatics analysis. This work was supported by JSPS KAKENHI (Grant number 19K09057).

\section{Supplementary Materials}

Figure S1: full sequences of HOXB9n and HOXB9v mRNA (black highlight indicates the homology between sequences). Figure S2: sequence results of PCR products of breast cancer cell-line derived genomic DNA. (Supplementary Materials)

\section{References}

[1] T. R. J. Lappin, D. G. Grier, A. Thompson, and H. L. Halliday, "HOX genes: seductive science, mysterious mechanisms," Ulster Medical Journal, vol. 75, no. 1, pp. 23-31, 2006.

[2] C. B. Moens and L. Selleri, "Hox cofactors in vertebrate development," Developmental Biology, vol. 291, no. 2, pp. 193-206, 2006.

[3] S. Bhatlekar, B. M. Fields, and B. M. Boman, "HOX genes and their role in the development of human cancers," Journal of Molecular Medicine, vol. 92, no. 8, pp. 811-823, 2014.

[4] R. Vikram, K. S. M. Ramachandran, and K. S. M. Abdul, "Functional significance of long non-coding RNAs in breast cancer," Breast Cancer, vol. 21, no. 5, pp. 515-521, 2014.

[5] D. X. Nguyen, A. C. Chiang, X. H.-F. Zhang et al., "WNT/TCF signaling through LEF1 and HOXB9 mediates lung adenocarcinoma metastasis," Journal of End-To-End-Testing, vol. 138, no. 1, pp. 51-62, 2009.
[6] O.-S. Kwon, E. Oh, J.-R. Park et al., "GalNAc-T14 promotes metastasis through Wnt dependent HOXB9 expression in lung adenocarcinoma," Oncotarget, vol. 6, no. 39, pp. 41916-41928, 2015.

[7] T. Hayashida, F. Takahashi, N. Chiba et al., "HOXB9, a gene overexpressed in breast cancer, promotes tumorigenicity and lung metastasis," Proceedings of the National Academy of Sciences, vol. 107, no. 3, pp. 1100-1105, 2010.

[8] B. Shrestha, K. I. Ansari, A. Bhan, S. Kasiri, I. Hussain, and S. S. Mandal, "Homeodomain-containing protein HOXB9 regulates expression of growth and angiogenic factors, facilitates tumor growth in vitro and is overexpressed in breast cancer tissue," The FEBS Journal, vol. 279, no. 19, pp. 3715-3726, 2012.

[9] Y. Hoshino, T. Hayashida, A. Hirata et al., "Bevacizumab terminates homeobox B9-induced tumor proliferation by silencing microenvironmental communication," Molecular Cancer, vol. 13, no. 1-14, p. 102, 2014.

[10] S. Y. Wu, R. Rupaimoole, F. Shen et al., "A miR-192-EGR1HOXB9 regulatory network controls the angiogenic switch in cancer," Nature Communications, vol. 7, no. 1, Article ID 11169, 2016.

[11] L. Sha, L. Dong, L. Lv, X. Bai, and X. Ji, "HOXB9 promotes epithelial-to-mesenchymal transition via transforming growth factor- $\beta 1$ pathway in hepatocellular carcinoma cells," Clinical and Experimental Medicine, vol. 15, no. 1, pp. 55-64, 2015.

[12] H. Seki, T. Hayashida, H. Jinno et al., "HOXB9 expression promoting tumor cell proliferation and angiogenesis is associated with clinical outcomes in breast cancer patients," Annals of Surgical Oncology, vol. 19, no. 6, pp. 1831-1840, 2012.

[13] A. Brock, S. Krause, H. Li et al., "Silencing HoxA1 by intraductal injection of siRNA lipidoid nanoparticles prevents mammary tumor progression in mice," Science Translational Medicine, vol. 6, no. 217, p. 217ra2, 2014.

[14] L. Fang, L. Xu, and L. Zou, "Overexpressed homeobox B9 regulates oncogenic activities by transforming growth factor$\beta 1$ in gliomas," Biochemical and Biophysical Research Communications, vol. 446, no. 1, pp. 272-279, 2014.

[15] C. Sun, C. Han, P. Wang, Y. Jin, Y. Sun, and L. Qu, "HOXB9 expression correlates with histological grade and prognosis in LSCC," BioMed Research International, vol. 2017, Article ID 3680305, 10 pages, 2017.

[16] J. Wan, H. Liu, Q. Feng, J. Liu, and L. Ming, "HOXB9 promotes endometrial cancer progression by targeting E2F3," Cell Death \& Disease, vol. 9, no. 5, p. 509, 2018.

[17] D. W. Huang, B. T. Sherman, Q. Tan et al., "DAVID bioinformatics resources: expanded annotation database and novel algorithms to better extract biology from large gene lists," Nucleic Acids Research, vol. 35, no. 2, pp. W169-W175, 2007.

[18] K. S. Keene, T. King, E. S. Hwang et al., "Molecular determinants of post-mastectomy breast cancer recurrence," Npj Breast Cancer, vol. 4, no. 1, p. 34, 2018.

[19] R. Morgan, A. Boxall, K. J. Harrington et al., "Targeting the HOX/PBX dimer in breast cancer," Breast Cancer Research and Treatment, vol. 136, no. 2, pp. 389-398, 2012.

[20] N. A. Laronde-leblanc and C. Wolberger, "Structure of HoxA9 and Pbxl bound to DNA: hox hexapeptide and DNA recognition anterior to posterior," Genes and Development, vol. 17, no. 16, pp. 2060-2072, 2003.

[21] H. D. Soule, T. M. Maloney, S. R. Wolman et al., "Isolation and characterization of a spontaneously immortalized human 
breast epithelial cell line, MCF-10," Cancer Research, vol. 50, no. 18, pp. 6075-6086, 1990.

[22] Y. Wang, J. Liu, B. Huang et al., "Mechanism of alternative splicing and its regulation," Biomedical Reports, vol. 3, no. 2, pp. 152-158, 2015.

[23] A. Nickless, J. M. Bailis, and Z. You, "Control of gene expression through the nonsense-mediated RNA decay pathway," Cell \& Bioscience, vol. 7, no. 1, p. 26, 2017.

[24] C. R. Stadler, N. Vegi, M. A. Mulaw et al., "The leukemogenicity of Hoxa9 depends on alternative splicing," Leukemia, vol. 28, no. 9, pp. 1838-1843, 2014.

[25] S. Fujimoto, K. Araki, O. Chisaka, M. Araki, K. Takagi, and K. I. Yamamura, "Analysis of the murine Hoxa-9 cDNA: an alternatively spliced transcript encodes a truncated protein lacking the homeodomain," Gene, vol. 209, no. 1-2, pp. 77-85, 1998.

[26] Y. S. Hong, S. Y. Kim, A. Bhattacharya, D. R. Pratt, W. K. Hong, and M. A. Tainsky, "Structure and function of the HOX A1 human homeobox gene cDNA," Gene, vol. 159, no. 2, pp. 209-214, 1995.

[27] W.-F. Shen, K. Detmer, T. A. Simonitch-Eason, C. Lawrence, and C. Largman, "Alternative splicing of the HOX 2.2 homeobox gene in human hematopoietic cells and murine embryonic and adult tissues," Nucleic Acids Research, vol. 19, no. 3, pp. 539-545, 1991.

[28] C. V. Wright, K. W. Cho, A. Fritz, E. M. Bürglin, and E. M. De Robertis, "A Xenopus laevis gene encodes both homeobox-containing and homeobox-less transcripts," The EMBO Journal, vol. 6, no. 13, pp. 4083-4094, 1987.

[29] N. Shah and S. Sukumar, "The Hox genes and their roles in oncogenesis," Nature Reviews Cancer, vol. 10, no. 5, pp. 361-371, 2010.

[30] H. Kocak, S. Ackermann, B. Hero et al., "Hox-C9 activates the intrinsic pathway of apoptosis and is associated with spontaneous regression in neuroblastoma," Cell Death \& Disease, vol. 4, no. 4, Article ID e586, 2013.

[31] R. Arya and K. White, "Cell death in development: signaling pathways and core mechanisms," Seminars in Cell \& Developmental Biology, vol. 39, pp. 12-19, 2015.

[32] K. Domsch, I. Papagiannouli, and I. Lohmann, "The HOXapoptosis regulatory interplay in development and disease," Current Topics in Developmental Biology, vol. 114, pp. 121158, 2015.

[33] P. M. J. In der Rieden, G. Mainguy, J. M. Woltering, and A. J. Durston, "Homeodomain to hexapeptide or PBC-interaction-domain distance: size apparently matters," Trends in Genetics, vol. 20, no. 2, pp. 76-79, 2004. 